\title{
Epigenetic Involvement in Hutchinson-Gilford Progeria Syndrome: A Mini-Review
}

\author{
Walter Arancio Giuseppe Pizzolanti Swonild I. Genovese Maria Pitrone \\ Carla Giordano \\ Section of Endocrinology, Diabetology and Metabolism, Biomedical Department of Internal and Specialized \\ Medicine (DiBiMIS), University of Palermo, Palermo, Italy
}

\section{Key Words}

Epigenetics · Hutchinson-Gilford progeria syndrome .

Ageing $\cdot$ Cellular senescence

\begin{abstract}
Hutchinson-Gilford progeria syndrome (HGPS) is a rare human genetic disease that leads to a severe premature ageing phenotype, caused by mutations in the LMNA gene. The $L M N A$ gene codes for lamin- $A$ and lamin- $C$ proteins, which are structural components of the nuclear lamina. HGPS is usually caused by a de novo C1824T mutation that leads to the accumulation of a dominant negative form of lamin-A called progerin. Progerin also accumulates physiologically in normal ageing cells as a rare splicing form of lamin-A transcripts. From this perspective, HGPS cells seem to be good candidates for the study of the physiological mechanisms of ageing. Progerin accumulation leads to faster cellular senescence, stem cell depletion and the progeroid phenotype. Tissues of mesodermic origin are especially affected by HGPS. HGPS patients usually have a bad quality of life and, with current treatments, their life expectancy does not exceed their second decade at best. Though progerin can be expressed in almost any tissue, when death occurs, it is usually due to cardiovascular complications. In HGPS, severe epigenetic alterations have been reported. Histone-covalent
\end{abstract}

modifications are radically different from control specimens, with the tendency to lose the bipartition into euchromatin and heterochromatin. This is reflected in an altered spatial compartmentalization and conformation of chromatin within the nucleus. Moreover, it seems that microRNAs and microRNA biosynthesis might play a role in HGPS. Exemplary in this connection is the suggested protective effect of miR-9 on the central nervous system of affected individuals. This mini-review will report on the state of the art of HGPS epigenetics, and there will be a discussion of how epigenetic alterations in HGPS cells can alter the cellular metabolism and lead to the systemic syndrome.

(c) 2014 S. Karger AG, Basel

\section{Hutchinson-Gilford Progeria Syndrome}

Progeroid syndromes are a group of systemic diseases that greatly resemble physiological ageing. Indeed, the term 'progeria' means 'prematurely old'. They usually refer to segmental progerias, where multiple tissues and organs are affected and patients usually show only some of the phenotypes and clinical conditions associated with ageing. They are all monogenic disorders that somehow alter either one of the DNA repair mechanisms or components of the nuclear lamina.

\section{KARGER}

E-Mail karger@karger.com

www.karger.com/ger
(C) 2014 S. Karger AG, Basel

0304-324X/14/0603-0197\$39.50/0
Walter Arancio, $\mathrm{PhD}$

DiBiMIS

Piazza delle Cliniche 2

IT-90127 Palermo (Italy)

E-Mail walter.arancio@unipa.it 
Hutchinson-Gilford progeria syndrome (HGPS) was named after Dr. Jonathan Hutchinson and Dr. Hastings Gilford, who first described it in England 1886 and 1897, respectively. It is a very rare disease, reported to occur in 1 in 8 million newborns worldwide. More than 130 cases have been reported in the scientific literature since the condition was first described [1].

HGPS phenotypes greatly resemble those that occur during natural ageing. One of the most striking differences is that HGPS is considered a developmental disease, as there is an improper development of tissues, especially of those of mesenchymal origin, before the development is complete [1].

HGPS is characterized by severe alterations of the nuclear architecture. Experimental data strongly suggest that these alterations may have a deep impact on the epigenetic state of the chromatin and in the regulation of transcription. Interestingly, the epigenetics of HGPS often resemble the epigenetics of normal ageing. It is still a matter of debate if and to what extent progeroid syndromes, especially HGPS, can be used as models of physiological ageing.

HGPS is a genetic condition characterized by the dramatic, rapid appearance of ageing beginning in childhood. Affected children typically look normal at birth and in early infancy, but then grow more slowly than unaffected children and experience what is commonly known as a 'failure to thrive.' They develop a typical facial appearance including prominent eyes, a thin nose with a beaked tip, thin lips, a small chin and protruding ears. Patients with HGPS also have complete alopecia, agedlooking skin, joint abnormalities and loss of subcutaneous fat. The condition does not disturb the intellectual development or the development of motor skills.

People with HGPS experience severe arteriosclerosis beginning in childhood. This condition greatly increases the chances of having a heart attack or stroke at a young age. These serious complications can worsen over time and are life-threatening. Children with HGPS typically die of heart disease at an average age of 13 years. HGPS is an autosomal dominant condition caused by de novo mutations in the LMNA gene that codes for nuclear lamins [1].

Lamins are nuclear intermediate filaments with structural and regulative functions, present in all metazoans. The absence of lamins in unicellular organisms is worth noting. Four major lamins are present in humans: lamin$\mathrm{B} 1$ and lamin-B2, coded from two independent loci, and lamin-A and lamin-C, coded by the same LMNA gene by alternative splicing.

Mutations in nuclear lamins cause a very heterogeneous class of diseases called laminopathies. The accu- mulation of a particular splicing form of lamin-A, called progerin, leads to the rare HGPS laminopathy. For an exhaustive review on lamins, please refer to Dechat et al. [2].

Usually, lamin-A is synthesized as a precursor of the mature form. The C-terminus of the protein undergoes multistep posttranslational processing. On its C-terminal $\mathrm{CaaX}$ motif, the precursor is farnesylated on cysteine, and then the remaining 3 amino acids are cleaved. The $\mathrm{C}$-terminal cysteine is then carboxymethylated. The farnesylated and carboxymethylated pre-lamin- $\mathrm{A}$ is then anchored into the inner nuclear membrane. Finally, the last $18 \mathrm{ami}$ no acids are cleaved by the metallopeptidase Zmpste24 to produce the mature, unfarnesylated lamin-A.

In HGPS, the proper synthesis and maturation of lamin-A is impaired. This is usually caused by a de novo autosomal dominant synonymous $\mathrm{G} 608 \mathrm{G}$ mutation within exon 11 of the LMNA gene (C1824T at the nucleotide level). This mutation activates a cryptic splicing site that leads to the production of a protein, called progerin, lacking 50 amino acids. This deletion abrogates the ZmpSte24 cleavage site, so progerin becomes constitutively farnesylated and anchored into the inner nuclear membrane [2].

A- and B-type lamins are expressed differently during differentiation. B-type lamins seem to be essential and ubiquitously expressed. By contrast, lamin-A production begins during differentiation and is maintained in fully differentiated cells [3]. It is to be noted that progerin seems to physiologically accumulate in cells as a function of the chronological age of donors and the number(s) of passages in culture. Progerin in nonmutated individuals is produced as a rare splicing form using a cryptic splicing site. Cells that accumulate progerin are prone to senescence, and animal models of HGPS recapitulate the progeroid characteristics of HGPS. From this perspective, HGPS cells are very good candidates for studying the physiological characteristics of human ageing. Interestingly, induced pluripotent stem cells from individuals affected by HGPS or from very old people are indistinguishable from those obtained from healthy donors. Intriguingly, as has been noted for embryonic stem cells, reprogrammed induced pluripotent stem cells do not express lamin-A [4]. This gives rise to the possibility that abrogating lamin-A expression may serve to treat both HGPS and physiological ageing.

At the cellular level, lamins can directly and indirectly bind to DNA in matrix attachment/scaffold-associated regions, though clear consensus sequences do not seem to be present. Lamin-associated regions tend to be heterochromatic and transcriptionally silent [5].

HGPS causes typical cellular phenotypes such as nuclear lobulation or blebbing - the cytological hallmark of 
HGPS - mitochondrial dysfunction [6], increased reactive oxygen species production [6] and chromosomal and telomere aberrations [7]. HGPS cells have altered cell-cycle regulation, impaired DNA repair mechanisms, a higher apoptosis rate and quicker cellular senescence [7].

The alteration of the cell cycle seems of particular interest; indeed, it has long been known that retinoblastoma protein is stabilized and regulated by nuclear lamina interactions [8]. Hence, the altered cell-cycle regulation observed in HGPS cells is probably due to an impaired function of the retinoblastoma protein network. Moreover, two recent in silico ceRNA and interactome analyses [9, 10] pointed to several other genes involved in the control of the cell cycle (TP53, MYC and E2F1 amongst many others) that might be deregulated in HGPS, but the significance of this is still to be investigated. Unexpectedly, the occurrence of cancer in HGPS patients is a very rare event $[1,3]$. This is one of the major differences between the HGPS phenotype and physiological ageing.

In HGPS, severe epigenetic alterations have been reported. Histone-covalent modifications, histone variants, DNA methylation, chromatin remodelers, chromatin architecture and miRNAs are all altered in HGPS. The epigenetic changes reported in HGPS and how these might lead to the cellular phenotype will be discussed in detail in this mini-review.

\section{Histone Modifications}

Profound alterations in the histone-covalent modifications of HGPS cells have been widely reported. These alterations concern both the type and spatial localization of the modification. Usually, nuclear lamina constitutes a repressive heterochromatic environment for the chromatin territories associated with it. In HGPS, there is a loss of peripheral heterochromatin and relative histone modifications (H3K9me3, H3K27me3) and associated proteins (HP1).

$\mathrm{H} 3 \mathrm{~K} 9 \mathrm{me} 3$ is one of the constitutive heterochromatin markers of the pericentric region and telomeres. It is downregulated in HGPS, and pericentric regions tend to be less associated with the nuclear lamina. As a consequence, pericentric transcription arises and the association with HP1, which recognizes $\mathrm{H} 3 \mathrm{~K} 9 \mathrm{me} 3$, decreases [2, $11]$.

$\mathrm{H} 3 \mathrm{~K} 27 \mathrm{me} 3$ is another heterochromatic marker that marks, for example, the $\mathrm{X}$-inactive chromosome in humans. In HGPS, it is downregulated, just like the H3K27 methyltransferase EZH2 [11].
The opposite behavior is shown for $\mathrm{H} 4 \mathrm{~K} 20 \mathrm{me} 3$, which is upregulated in HGPS cells. Interestingly, it marks telomeric heterochromatin, and an increase in H4K20me3 blocks telomere elongation. The data are consistent with the reported telomere dysfunction and accelerated senescence in HGPS cells [12]. H4K20 monomethylation also seems to increase in HGPS cells and acts as a marker of transcriptionally active chromatin [11, 13].

A detailed epigenome mapping analysis has revealed that there is a trend towards homogeneous redistribution of epigenetic markers in HGPS cells. As a consequence, the difference between high-expression genes and lowexpression genes tends to be minimized. Exemplary in this connection is $\mathrm{H} 3 \mathrm{~K} 27 \mathrm{me} 3$ (as a marker of facultative heterochromatin). Though it is downregulated as an absolute quantity in HGPS cells compared with control cells, the remnant $\mathrm{H} 3 \mathrm{~K} 27 \mathrm{me} 3$ is widely redistributed within HGPS chromatin. The trend reported is that $\mathrm{H} 3 \mathrm{~K} 27 \mathrm{me} 3$ decreases in gene-poor regions and increases in gene-rich regions. This correlates with the increased expression of poorly expressed genes and the decreased expression of highly expressed genes [14]. Interestingly, the same trend has been reported in senescent cells.

The data on histone methylation are quite robust; the role of acetylation in HGPS, however, is still controversial. Sirtuins are deacetylases that are also active on acetylated histone substrates, with a suggested role in regulating ageing processes. SIRT1 deacetylase activity is greatly enhanced by lamin-A association and is downregulated in senescent cells. Reported targets of SIRT1 are H4K16ac and $\mathrm{H} 3 \mathrm{~K} 9 \mathrm{ac}$. Consistently during ageing, H4K16ac is upregulated [15]. Opposite data have been retrieved in an HGPS mouse model, however, where histone H4K16 hypoacetylation was associated with premature senescence that might have been reversed by a histone acetyl transferase (HAT) Mof overexpression or by histone deacetylase inhibition [16]. Interestingly, in a recent in silico interactome analysis, a central role of histone acetylation has been proposed for the LMNA network, with a bias toward the histone deacetylase HTATIP (also known as KAT5 or TIP60), which is the catalytic subunit of the NuA4 histone acetyltransferase complex that preferably targets $\mathrm{H} 2 \mathrm{~A}$ and $\mathrm{H} 4$ histones [10].

Apart from methylation and acetylation, other histone-covalent modifications seem to be altered in HGPS. PARP1 [the poly (ADP-ribose) polymerase 1 gene] is a gene that has been linked to longevity in mammals [17]. PARP1 has a key role in regulating cell-cycle and DNA repair mechanisms. Moreover, histones and other chromatin proteins are the $P A R P 1$ substrates of choice. Interestingly, PARP1 is downregulated in HGPS cells [18]. 
Histone sumoylation also seems to be reduced in HGPS, with severe consequences in heterochromatin formation. Indeed, an increase in progerin is correlated to a reduced ratio between the nuclear and cytoplasmic Ran GTPase. This, in turn, inhibits the nuclear localization of Ubc9, the only known E2 required for sumoylation. Interestingly, usually both histones and chromatin remodelers tend to be deeply sumoylated [19]. Reduced sumoylation of histones and other nuclear proteins may be expected in HGPS cells.

\section{Histone Variants}

Histones are a major component of chromatin, fundamental to genome regulation. Histone variants lead to changes in chromatin dynamics and carry out specific functions. In addition, posttranslational modifications that occur on the variants may be different from those of canonical histones. Histone variants differ mostly in the $\mathrm{N}$-terminal region from their canonical counterpart. More than 50 unique histone variants are present in humans. The expression of histone variants can be replication dependent or replication independent. Histone variants can mark a specific region of the DNA by replacing canonical histones. It has been suggested that histone variant retention could be involved in epigenetic cellular memory.

In HGPS cells, a 6-fold downregulation of the mRNA for the H2A.X histone variant has been reported, often associated with a region of damaged DNA and a 2-fold downregulation of the mRNA for centromere-specific chromobox homolog 1 (CBX1) [18]. These data are consistent with the reported altered DNA repair mechanisms and centromere dynamics. It is interesting that HGPS cells are characterized by many unrepaired DNA doublestrand-break $\gamma$ H2A.X foci (marked by phosphorylated H2A.X) [20]; this poses interesting questions about the increased demand for and the decreased availability of H2A.X in HGPS cells. Of note, the accumulation of unrepaired DNA damage leads to the activation of ATM and ATR checkpoint kinases and cell-cycle arrest. It has been suggested that the accumulation of $\gamma \mathrm{H} 2 \mathrm{~A}$.X foci might be due to the lack of activity of the NuRD complex. The most striking characteristic of DNA damage-response impairment in HGPS cells is that $\gamma \mathrm{H} 2 \mathrm{~A}$.X foci fail to recruit Rad50 and Rad51 and instead recruit the Xeroderma pigmentosum group A protein (XPA), involved in the excision repair mechanism. It seems that the recruitment of XPA instead of Rad50 and $\operatorname{Rad} 51$ causes the failure of the double-strand-break repair mechanism [20]. This is a very rare, if not unique, example of a DNA repair mechanism that seems to impair the function of another.

An in silico ceRNA analysis also pointed to the involvement of the poorly studied H2AFY2 macroH2A histone variant [10]; a protective role in tumorigenesis and a role in ageing and $\mathrm{X}$ inactivation in mammals have been suggested for H2AFY2.

Interestingly, differences in the expression of core histones have also been reported. A proteomic study [21] showed a profound downregulation of histone $\mathrm{H} 4$ in HGPS cells compared to control cells. This poses an interesting and unanswered question on the composition of nucleosomes in HGPS cells lacking H4 core histones. Moreover, the same study [21] pointed to the downregulation of histone $\mathrm{H} 2 \mathrm{~B}$ types $2-\mathrm{E}, 1-\mathrm{M}$ and $1-\mathrm{C} / \mathrm{E} / \mathrm{F} / \mathrm{G} / \mathrm{I}$ in HGPS, which is of unknown biological significance.

\section{DNA Methylation}

Cytosine methylation on $\mathrm{CpG}$ islands on gene promoters is often involved in transcriptional silencing. In HGPS, the methylation profile is perturbed.

In a mouse model of HGPS, ribosomal RNA genes (rDNA) are hypermethylated. The same phenotype can be observed in ageing mice. The production of ribosomal RNA is consistently downregulated [22].

More recently, it has been observed that $\mathrm{CpG}$ sites in HGPS cells gain methylation when compared to hypomethylated control regions but lose methylation when compared to hypermethylated control regions [23]. Again, the trend is a homogeneous redistribution of epigenetic markers in HGPS specimens.

\section{ATP-Dependent Chromatin Remodelers}

ATP-dependent chromatin remodelers usually act in huge multiprotein complexes. The complexes are organized around a protein with ATPase activity. The energy from the hydrolysis of ATP allows these remodeling complexes to change the relative positioning of nucleosomes on the DNA string, to expel or exchange histones or to alter the local chromatin topology.

Specifically, a role for the NuRD complex has been proposed in HGPS. NuRD is a chromatin-remodeling complex with both ATP-dependent chromatin-remodeling and histone-deacetylase activities. Progerin lacks the amino-acidic sequence required to contact RBBP $4 / 7$ of the NuRD complex and, in turn, the NuRD complex ac- 
tivity seems to be impaired. Moreover, the interaction between lamin-A and NuRD is required to maintain a correct $\mathrm{H} 3 \mathrm{~K} 9$ methylation profile and the correct loading of heterochromatin protein 1 gamma (HP1 $\gamma)$ in chromatin. It has been suggested that these alterations might be the cause of the reported reduction in NuRD functions within a nuclear lamina context and the loss of peripheral heterochromatin in HGPS cells [24].

In addition, in silico analyses looking for attributes relevant to gene ontology in the LMNA network have pointed to the potential involvement of ATP-dependent chromatin remodelers. A more detailed analysis has revealed a bias towards the SMARCA4 (also known as BRG1), PBRM1 and SMARCB1 genes and pBAF and SWI/SNF complexes [10].

\section{Nuclear Architecture}

Chromosomes are nonrandomly organized into chromosomal territories. Gene-poor heterochromatic regions are preferably located closer to the nuclear lamina. Transcriptionally silent chromatin is usually in the interior of chromosomal territories, while transcriptionally active chromatin is located on the surface $[2,5,7]$. The nucleus is like a worm-eaten apple where the transcriptionally active euchromatin tends to be located towards the wormholes. In HGPS, the nuclear organization and the relative epigenetic markers are subverted.

In HGPS, the nuclear lamina is highly disorganized. Typically, progerin accumulation provokes a severe distortion of the nuclear lamina with typical blebbing that can be cytologically observed. This distortion of the lamina is associated with a severe loss of nuclear organization and nuclear compartmentalization $[2,5,7]$. It seems that the nuclear lamina is required for the correct organization of heterochromatin in the nuclear periphery. The accumulation of progerin strongly interferes with the metabolism of peripheral heterochromatin as well as with the whole nuclear architecture and heterochromatin/euchromatin spatial bipartition [14]. Indeed, in HGPS, there is a clear loss of peripheral heterochromatin and relative histone modifications (H3K9me3, H3K27me3) and associated proteins (HP1) [25]. In fact, heterochromatin tends to dissociate from the nuclear lamina, and there is a loss of spatial nuclear compartmentalization [14].

Several genes involved in the maintenance of the nuclear architecture are altered in HGPS. The cellular distribution of barrier-to-autointegration factor (BAF), a mitotic heterochromatin organization factor associated with the nuclear lamina, is profoundly altered in HGPS. Usually, BAF is present at both the nuclear and cytoplasmic levels, but in HGPS the cytoplasmic pool is severely depleted [26]. Interestingly, it has been reported that a mutation in BAF coded by the BANF1 gene leads to a progeroid phenotype [27].

In HGPS cells, the expression, function and nuclear localization of inhibitor of growth protein 1 (ING1) are impaired. ING1 is a tumor suppressor of the p53 pathway with a reported role in apoptosis and senescence. It usually has a bridge function between a methylated histone $\mathrm{H} 3$ and the acetylase/deacetylase complexes, and it is involved in the formation of peripheral heterochromatin [28].

The D4Z4 human subtelomeric repeats localize the telomeres to the nuclear periphery in a lamin-A-dependent manner [29]. This function is probably altered in HGPS.

High mobility group (HMG) proteins are able to bind and bend DNA double strands. The transcription of HMG-B2, HMG-17 and HMG-2 proteins is downregulated in HGPS cells [18]. The transcription of heterochromatic CBX1, also known as heterochromatin protein 1 homolog beta, is downregulated in HGPS cells [18]. It is a heterochromatic protein that recognizes and binds $\mathrm{H} 3 \mathrm{~K} 9 \mathrm{me} 3$, leading to epigenetic repression. It contributes to the association of heterochromatin with the inner nuclear membrane.

In silico analyses have also pointed to the involvement of $C B X 5$, which codes for HP1 $\alpha$, a heterochromatic protein that recognizes and binds $\mathrm{H} 3 \mathrm{~K} 9 \mathrm{me} 3$, leading to epigenetic repression [10].

\section{miRNAs}

miRNAs have multiple roles in negative and possibly in positive gene regulation. This regulation may be asserted at the transcriptional, posttranscriptional and translational levels. miRNAs are involved in most biological processes. The aberrant expression of miRNAs has been implicated in numerous pathological states.

Amongst the miRNAs, an important role for miR-9 has been suggested in HGPS. miR-9 is the only validated miRNA that interacts with the $3^{\prime}$ UTR of lamin-A/progerin $\mathrm{mRNA}$, and it plays a clear neuroprotective role in the brain. Lamin-A and progerin have the same $3^{\prime}$ UTR, but lamin-A and lamin-C have different $3^{\prime}$ UTRs. In a mouse model, it seems that the brain-specific miR-9 is able to strongly downregulate the production of lamin-A/progerin, leaving the level of lamin-C unchanged, via the inter- 
action with lamin-A/progerin $3^{\prime}$ UTRs. This might explain why the mouse models of HGPS are free of central nervous system pathology. A similar mechanism of protection could be hypothesized for humans, also with no involvement of the nervous system of HGPS patients [30].

Some interest has arisen about the miR-29 family. Indeed, in a mouse model of HGPS, the tumor suppressor miR-29 family is upregulated in response to DNA damage in a p53-dependent manner [31]. The authors of that study suggested that miR-29 may act as an ageing promoter, as many classic oncogenes are reported to be.

Interestingly, Arancio et al. [9] suggested a role for Dicer1, Argonaute1, Argonaute2 and Drosha (which have key activities in miRNA metabolism) and, to a lesser degree, Pasha (a partner of Drosha) in the lamin-A/ progerin network.

\section{Unanswered Questions}

There are still many questions that are waiting for an answer. The first is whether studies on HGPS might help to ameliorate the conditions of elderly people. There is an ongoing debate about the evolutionary ageing hypotheses [32] and on how HGPS could play a part here. Another question is whether or not HGPS studies might help to uncover the mechanism of ageing in order to slow it down, stop it or even reverse it. It is clear that in HGPS cells, the epigenetics are profoundly altered, and many efforts have been made to elucidate the changes in the patterns of histone methylation. Unanswered questions remain concerning the dynamics of histone acetylation. Many studies have pointed to a central role of acetylation/ deacetylation patterns and related modifying complexes in HGPS chromatin, but the data are not exhaustive and often controversial.

Interestingly, the reported downregulation of $P A R P 1$ and histone $\mathrm{H} 4$ might have a deep impact on chromatin dynamics, but there are almost no data on their role in HGPS.

Intriguingly, the downregulation of Hsp90 in HGPS cells has been reported [21] and a role for Hsp90 in the inhibition of mutagenic activity of endogenous retrotransposons has been demonstrated [33]. In the authors' opinion, these data taken together suggest a possible contribution of the reactivation of formally dormant transposable elements on the reported genomic instability in HGPS cells [20].

At least, the role of the histone variant $\mathrm{H} 2 \mathrm{~A}$.X is interesting. In HGPS, it is downregulated, but it is very much in demand in the attempt to repair putative multiple double-strand breaks at the level of $\gamma \mathrm{H} 2 \mathrm{~A}$.X foci. Here, once again, the double-strand-break repair mechanism is impaired due to the presence of the proteins of the excision repair mechanism in the $\gamma \mathrm{H} 2 \mathrm{~A}$.X foci. The biological significance of these events is not clear.

\section{Future Perspectives}

Experimental therapies rely on farnesylation inhibitors that turn progerin into a nonfarnesylated form that resembles the common lamin-A. Though these approaches seem to partially ameliorate the conditions of HGPS patients, an efficient cure is still to be developed.

Interestingly, in mouse models, it seems that only the expression of lamin- $\mathrm{C}$, and not that of lamin- $\mathrm{A}$, is compatible with life. The production of lamin-C only seems to occur physiologically in the mouse nervous system, thanks to the activity of miR-9 [30]. Strategies aimed at shifting the production from the LMNA gene towards lamin-C instead of lamin-A/progerin might soon be developed. A possible role of miR-9 in these strategies should be considered.

\section{Conclusions}

HGPS is a progeroid syndrome that seems to recapitulate many cellular and molecular characteristics of physiological ageing. How the accumulation of progerin leads to the phenotype is still under investigation. However, a clear change in the epigenetic landscape has been widely reported. In HGPS cells, there is a global loss of heterochromatic markers and an evident loss of asymmetry of epigenetic markers and levels of transcription amongst lowly and highly transcribed genes. This is associated with a partial loss of nuclear compartmentalization, which is probably due to the loss of the capacity of nuclear lamina to orchestrate the nuclear architecture via the involvement of the ATP-dependent chromatin remodeling complex NuRD amongst others.

At least there is increasing evidence of miRNA involvement in HGPS, and a suggested protective effect of miR-9 in the nervous system of affected individuals. A deeper understanding of how a disruption in the nuclear architecture could lead to such deep changes in chromatin dynamics and to an ageing phenotype might help to develop a healing strategy for progeroid syndromes and to ameliorate the condition of ageing humans. 


\section{References}

1 Gonzalez JM, Pla D, Perez-Sala D, Andres V: A-type lamins and Hutchinson-Gilford progeria syndrome: pathogenesis and therapy. Front Biosci (Schol Ed) 2011;3:1133-1146.

$>2$ Dechat T, Pfleghaar K, Sengupta K, Shimi T, Shumaker DK, Solimando L, Goldman RD: Nuclear lamins: major factors in the structural organization and function of the nucleus and chromatin. Genes Dev 2008;22:832-853.

$>3$ Zuela N, Bar DZ, Gruenbaum Y: Lamins in development, tissue maintenance and stress. EMBO Rep 2012;13:1070-1078.

4 Liu GH, Barkho BZ, Ruiz S, Diep D, Qu J, Yang SL, Panopoulos AD, Suzuki K, Kurian L, Walsh C, Thompson J, Boue S, Fung HL, Sancho-Martinez I, Zhang K, Yates J 3rd, Izpisua Belmonte JC: Recapitulation of premature ageing with iPSCs from HutchinsonGilford progeria syndrome. Nature 2011;472: 221-225.

$\checkmark 5$ Kubben N, Adriaens M, Meuleman W, Voncken JW, van Steensel B, Misteli T: Mapping of lamin A- and progerin-interacting genome regions. Chromosoma 2012;121:447464.

$\checkmark 6$ Rivera-Torres J, Acín-Perez R, CabezasSánchez P, Osorio FG, Gonzalez-Gómez C, Megias D, Cámara C, López-Otín C, Enríquez JA, Luque-García JL, Andrés V: Identification of mitochondrial dysfunction in HutchinsonGilford progeria syndrome through use of stable isotope labeling with amino acids in cell culture. J Proteomics 2013;91:466-477.

7 Prokocimer M, Barkan R, Gruenbaum Y: Hutchinson-Gilford progeria syndrome through the lens of transcription. Aging Cell 2013;12:533-543.

8 Johnson BR, Nitta RT, Frock RL, Mounkes L, Barbie DA, Stewart CL, Harlow E, Kennedy BK: A-type lamins regulate retinoblastoma protein function by promoting subnuclear localization and preventing proteasomal degradation. Proc Natl Acad Sci USA 2004;101: 9677-9682.

>9 Arancio W, Giordano C, Pizzolanti G: A ceRNA analysis on LMNA gene focusing on the Hutchinson-Gilford progeria syndrome. J Clin Bioinforma 2013;3:2.

10 Arancio W: A bioinformatics analysis of Lamin-A regulatory network: a perspective on epigenetic involvement in Hutchinson-Gilford progeria syndrome. Rejuvenation Res 2012;15:123-127.

11 Shumaker DK, Dechat T, Kohlmaier A, Adam SA, Bozovsky MR, Erdos MR, Eriksson M, Goldman AE, Khuon S, Collins FS, Jenuwein T, Goldman RD: Mutant nuclear lamin A leads to progressive alterations of epigenetic control in premature aging. Proc Natl Acad Sci USA 2006;103:8703-8708.
12 Cao K, Blair CD, Faddah DA, Kieckhaefer JE, Olive M, Erdos MR, Nabel EG, Collins FS: Progerin and telomere dysfunction collaborate to trigger cellular senescence in normal human fibroblasts. J Clin Invest 2011;121 2833-2844.

13 Barski A, Cuddapah S, Cui K, Roh TY, Schones DE, Wang Z, Wei G, Chepelev I, Zhao K: High-resolution profiling of histone methylations in the human genome. Cell 2007; 129:823-837.

14 McCord RP, Nazario-Toole A, Zhang H, Chines PS, Zhan Y, Erdos MR, Collins FS, Dekker J, Cao K: Correlated alterations in genome organization, histone methylation, and DNA-lamin A/C interactions in HutchinsonGilford progeria syndrome. Genome Res 2013;23:260-269.

15 Fraga MF, Esteller M: Epigenetics and aging: the targets and the marks. Trends Genet 2007; 23:413-418.

16 Krishnan V, Chow MZ, Wang Z, Zhang L, Liu B, Liu X, Zhou Z: Histone H4 lysine 16 hypoacetylation is associated with defective DNA repair and premature senescence in Zmpste24-deficient mice. Proc Natl Acad Sci USA 2011;108:12325-12330.

17 Mangerich A, Bürkle A: Pleiotropic cellular functions of PARP1 in longevity and aging: genome maintenance meets inflammation. Oxid Med Cell Longev 2012;2012:321653.

18 Ly DH, Lockhart DJ, Lerner RA, Schultz PG: Mitotic misregulation and human aging. Science 2000;287:2486-2492.

19 Kelley JB, Datta S, Snow CJ, Chatterjee M, Ni L, Spencer A, Yang CS, Cubeñas-Potts C, Matunis MJ, Paschal BM: The defective nuclear lamina in Hutchinson-gilford progeria syndrome disrupts the nucleocytoplasmic Ran gradient and inhibits nuclear localization of Ubc9. Mol Cell Biol 2011;31:3378-3395.

20 Hutchison CJ: The role of DNA damage in laminopathy progeroid syndromes. Biochem Soc Trans 2011;39:1715-1718.

21 Wang L, Yang W, Ju W, Wang P, Zhao X, Jenkins EC, Brown WT, Zhong N: A proteomic study of Hutchinson-Gilford progeria syndrome: application of 2D-chromotography in a premature aging disease. Biochem Biophys Res Commun 2012;417:1119-1126.

22 Osorio FG, Varela I, Lara E, Puente XS, Espada J, Santoro R, Freije JM, Fraga MF, LópezOtín C: Nuclear envelope alterations generate an aging-like epigenetic pattern in mice deficient in Zmpste24 metalloprotease. Aging Cell 2010;9:947-957.
23 Heyn H, Moran S, Esteller M: Aberrant DNA methylation profiles in the premature aging disorders Hutchinson-Gilford progeria and Werner syndrome. Epigenetics 2013;8:28-33.

24 Meshorer E, Gruenbaum Y: NURD keeps chromatin young. Nat Cell Biol 2009;11: 1176-1177.

25 Simboeck E, Ribeiro JD, Teichmann S, Di Croce L: Epigenetics and senescence: learning from the INK4-ARF locus. Biochem Pharmacol 2011;82:1361-1370.

26 Capanni C, Cenni V, Haraguchi T, Squarzoni S, Schüchner S, Ogris E, Novelli G, Maraldi N, Lattanzi G: Lamin A precursor induces barrier-to-autointegration factor nuclear localization. Cell Cycle 2010;9:2600-2610.

-27 Puente XS, Quesada V, Osorio FG, Cabanillas R, Cadiñanos J, Fraile JM, Ordóñez GR, Puente DA, Gutiérrez-Fernández A, FanjulFernández M, Lévy N, Freije JM, López-Otín $\mathrm{C}$ : Exome sequencing and functional analysis identifies BANF1 mutation as the cause of a hereditary progeroid syndrome. Am J Hum Genet 2011;88:650-656.

28 Han X, Feng X, Rattner JB, Smith H, Bose P, Suzuki K, Soliman MA, Scott MS, Burke BE, Riabowol K: Tethering by lamin A stabilizes and targets the ING1 tumour suppressor. Nat Cell Biol 2008;10:1333-1340.

29 Ottaviani A, Schluth-Bolard C, Rival-Gervier S, Boussouar A, Rondier D, Foerster AM, Morere J, Bauwens S, Gazzo S, Callet-Bauchu E, Gilson E, Magdinier F: Identification of a perinuclear positioning element in human subtelomeres that requires A-type lamins and CTCF. EMBO J 2009;28:2428-2436.

30 Jung HJ, Coffinier C, Choe Y, Beigneux AP, Davies BS, Yang SH, Barnes RH 2nd, Hong J, Sun T, Pleasure SJ, Young SG, Fong LG: Regulation of prelamin A but not lamin C by miR-9, a brain-specific microRNA. Proc Natl Acad Sci USA 2012;109:E423-E431.

31 Ugalde AP, Ramsay AJ, de la Rosa J, Varela I, Mariño G, Cadiñanos J, Lu J, Freije JM, López-Otín C: Aging and chronic DNA damage response activate a regulatory pathway involving miR-29 and p53. EMBO J 2011;30: 2219-2232.

-32 Trindade LS, Aigaki T, Peixoto AA, Balduino A, Mânica da Cruz IB, Heddle JG: A novel classification system for evolutionary aging theories. Front Genet 2013;4:25.

33 Specchia V, Piacentini L, Tritto P, Fanti L, D’Alessandro R, Palumbo G, Pimpinelli S, Bozzetti MP: Hsp90 prevents phenotypic variation by suppressing the mutagenic activity of transposons. Nature 2010;463:662-665. 